\title{
Socio-Environmental Patterns Associated with Cancer Mortality: A Study Based on a Quality of Life Approach
}

\author{
Sonia Alejandra Pou ${ }^{1,2}$, Maria del Pilar Diaz ${ }^{1,2 *}$, Guillermo Angel Velazquez ${ }^{3}$
}

\begin{abstract}
Background: With $18.6 \%$ of total deaths due to malignant tumors in 2016, cancer is the second leading death cause in Argentina. While there is a broad consensus on common risk factors at the individual cancer level, those operating at a contextual level have been poorly studied in developing countries. The objective of our study was to identify socio-environmental patterns in Argentina (2010), emphasizing quality of life, and to explore their associations with the spatial distribution of cancer mortality in the country. Methods: The study was conducted in 525 geographical divisions nested into 24 provinces. Sex-specific crude and age-standardized mortality rates (ASMR) for cancer (2009-2011 period) were calculated. Empirically derived socio-environmental patterns were identified through principal-component factor analysis on a selected set of variables: an urban scale and 29 indicators of a quality of life index in Argentina for 2010. Two-level Poisson regression models were used to estimate associations between the ASMR and the continuous factor scores for socio-environmental patterns as covariates. A random intercept was included to account for spatial variability in the ASMR distribution using Stata software. Results: Four socio-environmental patterns were identified, termed "Contexts with urban-related resources or cultural capital", "Socioeconomically prosperous contexts", "Environments with anthropic exposures" and "Plains region" (cumulative explained variance=57\%). High mortality rates were found in counties characterized by socioeconomically prosperous contexts $(\mathrm{RR}=1.025$ in women; 1.088 in men) and plain landscapes ( $R R=1.057$ and 1.117 , respectively). Counties featuring urban or cultural resources demonstrated increased mortality in women $(\mathrm{RR}=1.015,95 \% \mathrm{CI}=1.005-1.025)$, whereas rising rates associated with environments having anthropic exposures $(\mathrm{RR}=1.008,95 \% \mathrm{CI}=1.001-1.016)$ were observed only for men. Conclusion: This study identified four characteristic socio-environmental patterns in Argentina which incorporate features of quality of life, accounting to some extent for the differential burden of cancer mortality in this country.
\end{abstract}

Keywords: Neoplasms- epidemiology- quality of life - spatial analysis- Argentina

Asian Pac J Cancer Prev, 19 (11), 3045-3052

\section{Introduction}

Over the last decades, Argentina has been following the worldwide epidemiological trend toward rapid increases in the relative burden of non-communicable diseases. According to vital statistics registries, cancer is the second most common cause of death in this country $(18.6 \%$ of the total deaths in 2016 by National Cancer Institute), although there is wide geographical variation in cancer mortality burden across the territory with differences between social groups as well (Matos et al., 1994; Díaz et al., 2009; Pou et al., 2017). It has been proposed that the description of the geographic distribution of cancer statistics in Latin American countries identifies the profile of the population and, consequently, its quality of life standards (Curado and de Souza, 2014).

Cancer is characterized by uncontrolled cellular growth as a result of changes in the genetic information of cells, mostly related to genetic alterations or damages linked to both endogenous and exogenous factors and accumulated over time. Even so, based on the recognition of the complex, multifactorial and not fully understood etiology of this disease, in the field of cancer epidemiology there is renewed interest in approaching human health from a context perspective. This approach goes beyond the individual level adopted in classical biomedical research, which focuses on the effect of recognized behavioral - individual - risk factors of non-communicable diseases (such as smoking, physical inactivity and unhealthy diet). From a more integrative perspective, structural and intermediary determinants of people's health (including the socioeconomic and political context, social hierarchy, and environmental and behavioral factors) impact on people's risk of illness and

${ }^{I}$ Institute of Health Sciences Research (INICSA), Faculty of Medical Sciences, National Scientific and Technical Research Council (CONICET), ${ }^{2}$ Biostatistics Unit, School of Nutrition, Faculty of Medical Sciences, University of Córdoba, ${ }^{3}$ Institute of Geography, History and Social Sciences (IGEHCS), National Scientific and Technical Research Council (CONICET), National University of the Center of the Province of Buenos Aires, Tandil, Argentina.*For Correspondence: pdiaz@fcm.unc.edu.ar 
opportunities to cope with it (Solar and Irwin, 2010). We argue that the degree of exposure of people to exogenous factors (potentially carcinogenic) and the heath care opportunities are conditioned for population features related to its quality of life. These include the social and physical contexts in which they live, where we may find the "upstream" determinants of cancer burden distribution.

Up to now, most epidemiological studies on cancer and quality of life have been focused on the so-called "health-related quality of life", which is used to evaluate of the impact of treatment from the patient's perspective (Ghislain et al., 2016; Wang et al., 2016). Other works analyze, separately, cancer-related factors associated with well-being of populations, such as socioeconomic status or human development, and occupational exposures (Lagiou et al., 2005; Alavanja et al., 2013; Sierra et al., 2016). In this sense, a recent study conducted in Argentina reported significant associations between both poverty and urbanization and cancer burden (Pou et al., 2017). Interestingly, it was also showed the existence of high level of regional inequality in terms of a quality of life index and urban hierarchy in this country (Velázquez, 2016). To our knowledge, the present work is the first study that analyzes the link between the cancer mortality and people's quality of life in Argentina using a composite index at population level that integrates both the environmental and socioeconomic dimensions. This allowed us to obtain reliable and synthetic information about health inequities in cancer from a spatial perspective.

Considering that it has been highlighted that multilevel statistical and spatial approaches are useful to more accurately represent the geographical imprints of diverse contextual factors and the levels at which they influence cancer outcomes (Zahnd and McLafferty, 2017), two-level Poisson regression models (Rabe-Hesketh and Skrondal, 2012) were performed to describe expected values of cancer mortality rate related to socio-environmental covariates in Argentina, taking into account their spatial variability.

Thus, the objective of our study was to identify socioenvironmental patterns in Argentina (2010), emphasizing quality of life, and to explore their associations with the spatial distribution of cancer mortality in the country.

\section{Materials and Methods}

\section{Study Design and Data}

An ecological study was conducted in Argentina, using 525 geographical divisions (counties or communes) nested into 24 provinces ( 23 plus the Autonomous City of Buenos Aires).

Sex-specific crude mortality rates (CMR) and age-standardized mortality rates (ASMR) of cancer (codes C00-C97 in ICD-10th revision), per 100,000 persons/year, were calculated by the direct method (world standard population as reference) from 2009 up to 2011.

The 2010 Quality of Life Index (QoLI) by Velázquez (2016) was used, which incorporates the latest available census data (October 2010) into a Geographic Information System, also considering other national data sources (such as the Health and Wellbeing Ministry of Argentina reports, municipal information, field studies, satellite imagery). Basically, the index is conceived on two main dimensions: a) a socioeconomic one, which embraces indicators related to education, health and housing, and b) an environmental dimension, which refers to environmental problems as well as to the degree of attraction of "nature-based" landscapes and "socially constructed" amenities. Details of the calculation of the index have been published elsewhere (Velázquez, 2016).

In the present study, the urban scale of each county was also considered. It included six categories: from rural population of less than 2,000 inhabitants to large cities of $1,000,000$ or more inhabitants, based on the largest urban agglomeration within each geographic division in 2010 .

This research was conducted according to the principles of the Declaration of Helsinki. No ethical approval was required as it involved anonymized records and datasets existing in the public domain.

\section{Statistical Analysis}

Firstly, tendencies in CMR and ASMR distribution were explored by calculating the mean (SD) rates by tertile of each QoLI dimension and the index as a whole (Table 1).

Empirically derived Socio-environmental Patterns (SEPs) were identified through Factor Analysis (Principal Components Method with varimax rotation) (Johnson and Wichern, 2004) on a selected set of 30 variables: the urban scale and all indicators from the socioeconomic and environmental dimensions of the 2010 QoLI (see footnote in Table 2).

The Factor Analysis allows us to describe the variance-covariance structure among the variables as a synthetic number of underlying factors, referred to as SEP. The Kaiser-Meyer-Olkin (KMO) index and Bartlett's sphericity test were estimated for checking sampling adequacy and assumptions. Four factors were retained considering eigenvalue $>1$. Variables with rotated factor loadings $>0.60$ were considered to be "dominant", and were used to label the factors. It should be noted that for socioeconomic variables, the increment of its partial index number implies a better relative situation, while high factor loading for environmental problems represent a worse relative situation.

Scoring coefficients calculated by the regression method indicate the degree to which each county's socioenvironmental context conforms to one of the identified SEPs. The factor scores of each SEP were then incorporate into a Geographic Information System to map their geographical distribution (spatial patterns). The ArcMap central application of ArcGIS 10.2.2 software (Esri Inc. 1999-2014, US) was used.

Finally, multilevel (two-level) Poisson regression models (Rabe-Hesketh and Skrondal, 2012) were used to estimate association measures between the 2009-2011 mortality rates (three-year average) and the continuous factor scores of SEPs as covariates. Separate models for each sex were fitted using a random intercept to account for the spatial variability of ASMR distribution $(i=525$ counties nested into $j=24$ provinces). The linear predictor of proposed model was: 
$\log \left[\mathrm{E}\left(\mathrm{y}_{\mathrm{ij}}\right)\right]=\zeta_{\mathrm{j}}+\beta_{1} \mathrm{x}_{1 \mathrm{ij}}+\ldots+\beta_{4} \mathrm{x}_{4 \mathrm{ij}}$

where $y_{i j}$ is the response variable (ASMR for cancer), $\mathrm{x}_{1 \mathrm{ij}}$ to $\mathrm{x}_{4 \mathrm{ij}}$ are explanatory variables with fixed effects (linear coefficients), corresponding to the continuous factor scores of each SEP, and $\zeta_{\mathrm{j}}$ is a random intercept term, representing the clustering structure, with $\zeta_{j} \sim \mathrm{N}(0$, $\sigma^{2}$ ). The modeling assumptions were verified by using likelihood ratio test (Poisson regression nested into two-level Poisson model). Akaike Information Criterion (AIC) was used for comparison of non-nested models. All analyses were performed using Stata v14 software (StataCorp LP 1985-2013, USA).

\section{Results}

Cancer accounted for 123 and 157 deaths (per 100,000 persons/year) in Argentine women and men, respectively, in 2009-2011 period, with a ASMR around 31\% higher in the male than in the female population (Table 1). For both sexes, counties with the lowest QoLI (tertile I) also presented the lowest CMR or ASMR average. There were higher CMRs and ASMRs in the counties classified in the upper tertile of the socioeconomic QoLI group (versus the lower tertile), whereas for the environmental QoLI the lowest mortality rates corresponded to counties presenting the highest index values (except for female ASMR) (Table 1).

Figure 1 shows the spatial distribution of the QoLI. A higher value was found in the central-eastern area and counties located in southern Argentina. These regions also show higher socioeconomic index (better relative situations). Instead, according to the environmental QoLI, counties located in the western region presented the most favorable environmental indexes (Figure 1).

Table 2 shows the factor-loading matrix for the identified patterns (four retained factors). These factors explained $57 \%$ of the total variance in the original dataset. The Kaiser-Meyer-Olkin (KMO) measure of sampling adequacy for this dataset was 0.84 (meritorious: 0.80 to 0.89 ). The null hypothesis that the correlation matrix is an Identity one was rejected on the basis of Bartlett's test of sphericity $(\mathrm{p}<0.001)$.

The identified SEPs (Table 2, Figure 2) were called: 1) "Contexts with urban-related resources or cultural capital" (a high score for the urban scale, socially constructed recreational resources and the university degree indicator), 2) "Socioeconomically prosperous contexts" (a high score for several housing, health and education indexes), 3) "Environments with anthropic exposures" (a high score for environmental problems related to social interventions or activities) and 4) "Plains region" (negative scores for relief and seismicity/volcanism indicators). Figure 2 illustrates the spatial distributions of the continuous factor scores for each SEP, the magnitudes of which describe the degree of adherence to the pattern. Worthy of note is the regional distribution of SEP 2 and 4 (Figure 2-B and 2-D), concentrated in the central-eastern region.

The small AIC estimated values were obtained including the four SEPs as covariates in models (AIC $=7109.1$ and 7134.7 for female and men respectively). Significant positive associations $(\mathrm{p}<0.05)$ between cancer mortality rates and SEP $1(\mathrm{RR}=1.015)$, SEP $2(\mathrm{RR}=1.025)$, and SEP $4(R R=1.057)$ were found for women (Table 3). On the other hand, SEP $2(\mathrm{RR}=1.088), 3(\mathrm{RR}=1.008)$ and $4(\mathrm{RR}=1.117)$ were associated with increased cancer mortality rates in males.

Table 1. Crude and Age-Standardized Cancer Mortality Rates (2009-2011) in the Female and Male Populations of Argentina ( $\mathrm{n}=525$ counties): average (SD) by tertile of the quality of life index distribution and its dimensions. Argentina, 2010

\begin{tabular}{|c|c|c|c|c|}
\hline & \multicolumn{2}{|c|}{$\begin{array}{c}\text { Female population } \\
\text { Average }(\mathrm{SD}) \text { of cancer rates }\end{array}$} & \multicolumn{2}{|c|}{$\begin{array}{c}\text { Male population } \\
\text { Average (SD) of cancer rates }\end{array}$} \\
\hline & CMR & ASMR & CMR & ASMR \\
\hline & $122.86(52.19)$ & $88.55(25.53)$ & $156.99(67.79)$ & $128.52(37.53)$ \\
\hline \multicolumn{5}{|c|}{ QoLI 2010} \\
\hline \multicolumn{5}{|c|}{ Tertiles } \\
\hline I & $90.83(39.63)$ & $85.01(32.98)$ & $118.27(48.76)$ & $116.22(39.79)$ \\
\hline II & $128.36(50.06)$ & $90.04(23.96)$ & $166.26(70.01)$ & $130.58(38.47)$ \\
\hline III & $149.39(48.53)$ & $90.59(16.76)$ & $186.45(63.98)$ & $138.75(30.29)$ \\
\hline \multicolumn{5}{|c|}{ Socioeconomic QoLI } \\
\hline \multicolumn{5}{|c|}{ Tertiles } \\
\hline I & $88.40(36.86)$ & $83.87(31.26)$ & 113.04 (46.69) & $111.41(39.71)$ \\
\hline II & $116.08(40.63)$ & $89.22(25.41)$ & $147.06(55.67)$ & $127.59(35.54)$ \\
\hline III & $164.11(47.34)$ & $92.56(17.34)$ & $210.89(59.98)$ & $146.55(27.91)$ \\
\hline \multicolumn{5}{|c|}{ Environmental QoLI } \\
\hline \multicolumn{5}{|c|}{ Tertiles } \\
\hline I & $121.30(59.11)$ & $85.08(30.53)$ & $167.95(74.95)$ & $134.06(42.24)$ \\
\hline II & $135.69(52.02)$ & $93.17(23.16)$ & $170.04(68.21)$ & $132.21(34.74)$ \\
\hline III & $111.59(41.31)$ & $87.40(21.41)$ & $132.99(51.95)$ & $119.27(33.46)$ \\
\hline
\end{tabular}

SD, standard deviation; CMR, crude mortality rate; ASMR, age-standardized mortality rate; QoLI, quality of Life Index. 
A. QoLI 2010

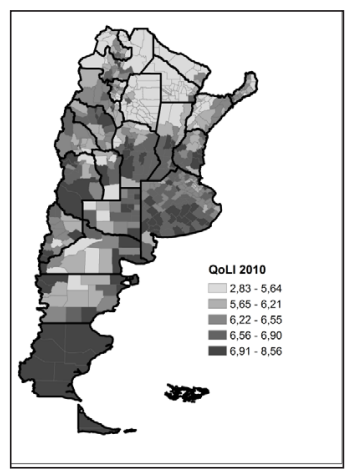

B. Socioeconomic QoLI

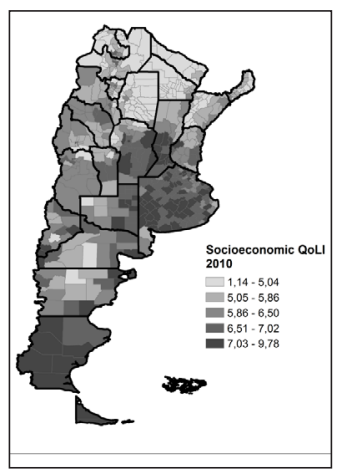

C. Environmental QoLI

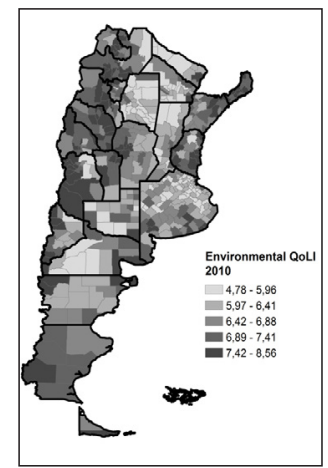

Figure 1. Spatial Distribution Maps of the Quality of Life Index (A) and Its Socioeconomic (B) and Environmental (C) Dimensions. Argentina, 2010. QoLI, quality of life index. Note: The increasing gradient of gray reflects the increment from lowest to highest quintile of the index distribution.

Table 2. Factor-Loading Matrix for the Four Socio-Environmental Patterns (SEP) Identified by the Principal Component Factor Analysis. Loadings greater than 0.60 (in absolute value) are shown in bold typeface, indicating the main characteristics of each factor.

\begin{tabular}{|c|c|c|c|c|}
\hline Indicator & SEP $1^{\mathrm{a}}$ & SEP $2^{b}$ & ${\text { SEP } 3^{c}}$ & SEP $4^{\mathrm{d}}$ \\
\hline Urban scale & 0.6538 & 0.0952 & 0.6015 & 0.1575 \\
\hline Beaches $^{e}$ & 0.1206 & 0.2026 & -0.1759 & 0.1208 \\
\hline Resorts by streams, rivers and lakes ${ }^{\mathrm{e}}$ & 0.0486 & 0.1173 & -0.2842 & 0.3021 \\
\hline Natural spas ${ }^{\mathrm{e}}$ & 0.1613 & -0.0512 & -0.1912 & 0.0122 \\
\hline Presence of ice and snow for winter activities ${ }^{\mathrm{e}}$ & 0.2496 & 0.0654 & -0.2604 & -0.4985 \\
\hline Relief $^{\mathrm{e}}$ & -0.0518 & -0.068 & -0.1081 & -0.8122 \\
\hline Lakes and streams ${ }^{\mathrm{e}}$ & 0.3474 & 0.0747 & -0.0667 & 0.2165 \\
\hline Parks and green open spaces ${ }^{\mathrm{e}}$ & 0.2217 & -0.3987 & -0.1095 & 0.4777 \\
\hline Urban aesthetics/urban heritage ${ }^{\mathrm{f}}$ & 0.8877 & 0.1663 & -0.0682 & -0.1 \\
\hline Cultural centers ${ }^{\mathrm{f}}$ & 0.9274 & 0.0949 & 0.1728 & -0.023 \\
\hline Shopping malls and other amenities ${ }^{\mathrm{f}}$ & 0.9377 & 0.1041 & 0.1984 & -0.0247 \\
\hline Sports centers ${ }^{\mathrm{f}}$ & 0.9369 & 0.1132 & 0.1489 & 0.0121 \\
\hline Use of pesticides in agriculture ${ }^{g}$ & -0.1116 & 0.179 & -0.133 & 0.5773 \\
\hline Participation of industry and mining in GNPg & 0.126 & 0.287 & 0.6334 & 0.171 \\
\hline Pollution/noise/traffic ${ }^{\mathrm{g}}$ & 0.4865 & 0.073 & 0.7076 & 0.1274 \\
\hline Hazardous locations $s^{g}$ & 0.3515 & 0.126 & 0.6786 & -0.0398 \\
\hline Locations with negative externalities ${ }^{\mathrm{g}}$ & 0.3242 & 0.0866 & 0.6775 & 0.098 \\
\hline Crime rate every 10,000 inhabitants $^{g}$ & 0.5599 & 0.178 & 0.073 & -0.3314 \\
\hline Seismicity and volcanism ${ }^{g}$ & 0.0936 & -0.1487 & -0.1219 & -0.8144 \\
\hline Tornadoes $^{\mathrm{g}}$ & -0.0456 & 0.5498 & 0.1419 & 0.6035 \\
\hline Flooding & -0.1695 & -0.3563 & 0.1922 & 0.3687 \\
\hline Percentage of the population living in slums ${ }^{g}$ & 0.3381 & -0.5827 & 0.243 & -0.0086 \\
\hline Percentage of the population living near dumps (less tah 300 meters) ${ }^{g}$ & 0.1979 & -0.4993 & 0.3916 & -0.1963 \\
\hline Climatic discomfortg & -0.0016 & -0.4576 & -0.5016 & -0.2509 \\
\hline Index of the population with an incomplete primary education ${ }^{\mathrm{h}, \mathrm{k}}$ & 0.439 & 0.708 & 0.3293 & -0.0764 \\
\hline Index of the population with a university degree $e^{\mathrm{h}, \mathrm{k}}$ & 0.6279 & 0.4274 & 0.24 & -0.0264 \\
\hline Index of the population without a health insurance or private or a mutual health plan ${ }^{\mathrm{i}, \mathrm{k}}$ & 0.2927 & 0.8468 & 0.1404 & 0.0443 \\
\hline Index of Infantile Mortality Rate according to the mother's residence place ${ }^{\mathrm{i}, \mathrm{k}}$ & 0.1408 & 0.4844 & -0.0742 & -0.1091 \\
\hline Index of the population living in houses without private toilets ${ }^{\mathrm{j}, \mathrm{k}}$ & 0.2823 & 0.7233 & 0.1343 & 0.1584 \\
\hline Index of the population in crowded homes ${ }^{\mathrm{j}, \mathrm{k}}$ & 0.2504 & 0.8311 & 0.0407 & 0.1648 \\
\hline Proportion of explained variances (\%) & 0.2729 & 0.1331 & 0.0995 & 0.0603 \\
\hline Cumulative explained variances (\%) & 0.2729 & 0.406 & 0.5055 & 0.5658 \\
\hline
\end{tabular}

SEP, socio-environmental pattern; GNP, Geographical National Product. a, SEP called "Contexts with urban-related resources or cultural capital; b, SEP called "Socioeconomically prosperous contexts"; ', SEP called "Environments with anthropic exposures"; d, SEP, called "Plains region". Indicator types according to the Quality of Life Index 2010 criterion; ${ }^{\mathrm{e}}$, Nature-based recreational resources; ${ }^{\mathrm{f}}$, Socially constructed recreational resources; ${ }^{\mathrm{g}}$, Environmental problems; ${ }^{\mathrm{h}}$, Educational; ${ }^{\mathrm{i}}, \mathrm{Health} ;{ }^{\mathrm{j}}$, Housing. ${ }^{\mathrm{k}}, \mathrm{A}$ high score value indicates a better relative situation. 
Table 3. Relative Risks (RRs) of Cancer ASMR, and 95\% Confidence Intervals, on Continuous Factor Scores of Socio- Environmental Patterns ( $\mathrm{n}=525$ counties). Argentina, 2010.

\begin{tabular}{lcccc}
\hline & \multicolumn{2}{c}{$\begin{array}{c}\text { Model I } \\
\text { Female population }\end{array}$} & \multicolumn{2}{c}{$\begin{array}{c}\text { Model II } \\
\text { Male population }\end{array}$} \\
Measures of association & RR $(95 \% \mathrm{CI})$ & $\mathrm{p}$ value & RR $(95 \% \mathrm{CI})$ & $\mathrm{p}$ value \\
\hline Covariates (continuous factor scores of SEP): & & & & \\
$\quad$ Contexts with urban-related resources or cultural capital & $1.015(1.005 ; 1.025)$ & $0.003^{*}$ & $1.004(0.996 ; 1.012)$ & 0.282 \\
Socioeconomically prosperous contexts & $1.025(1.016 ; 1.035)$ & $0.001^{*}$ & $1.088(1.080 ; 1.097)$ & $0.001^{*}$ \\
Environments with anthropic exposures & $1.002(0.992 ; 1.011)$ & 0.703 & $1.008(1.001 ; 1.016)$ & $0.045^{*}$ \\
Plains region & $1.057(1.046 ; 1.068)$ & $0.001^{*}$ & $1.117(1.108 ; 1.126)$ & $0.001^{*}$ \\
\hline Measures of clustering & Variance (SE) & & Variance (SE) & \\
\hline Intercept (counties) & $0.013(0.001)$ & & $0.014(0.001)$ & \\
\hline
\end{tabular}

RR, relative risk; CI, confidence interval; SEP, socio-environmental patterns; SE, standard error; Likelihood Ratio test vs. Poisson model; $\chi^{2}$ $(p$ value $)={ }^{a} 673.70(<0.001) ;{ }^{b} 785.15(<0.001) ; * A p$ value less than 0.05 was considered statistically significant.

A. Contexts with urban-related resources or cultural capital

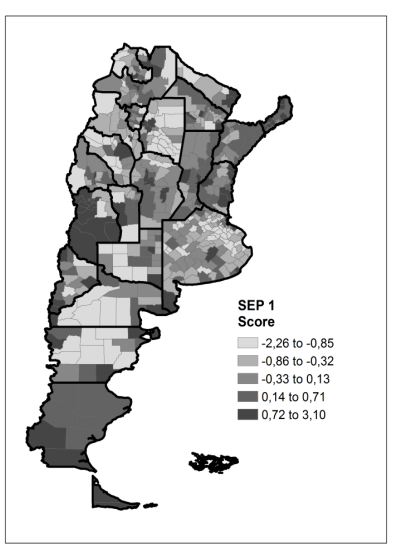

C. Environments with anthropic exposures

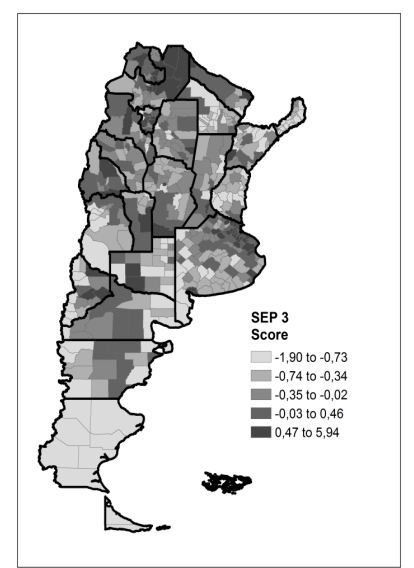

B. Socioeconomically prosperous contexts

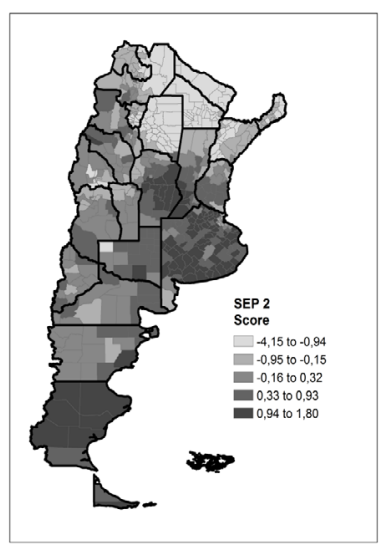

D. Plains region

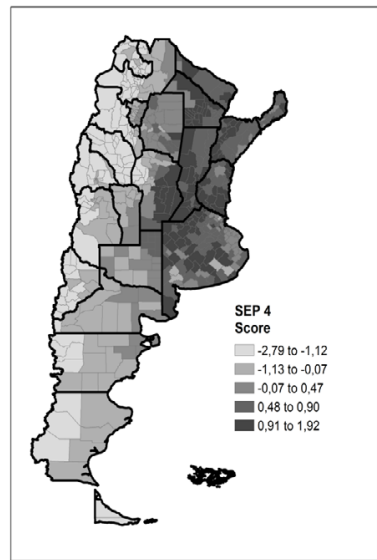

Figure 2. Maps of Distribution of Socio-Environmental Pattern (SEP) Factor Scores. Argentina, 2010. Figures 2-A, 2-B, 2-C and 2-D illustrate the distribution of the (continuous) factor score for the socio-environmental patterns 1 , 2,3 and 4, respectively, obtained by the factor analysis. The increasing gradient of gray reflects the increment from lowest to highest quintile of each factor score distribution, which in turn indicates the degree of adherence to each SEP.

\section{Discussion}

This study identified four characteristic socioenvironmental patterns in Argentina which incorporate features of quality of life and urbanization at county level, accounting for the differential burden of cancer mortality in this country. Results show that counties characterized by socioeconomically prosperous contexts or plains were associated with increased cancer ASMR for each sex. Moreover, contexts with urban-related resources or 
cultural capital were directly related to female ASMR, while areas with a higher anthropic environmental exposure presented higher male ASMR.

In this study, the estimated cancer mortality rates were higher in men than in women, which is consistent with other reported data about mortality statistics in Argentina (Ferlay et al., 2013; Sierra et al., 2016; Pou et al., 2017). Some authors suggested that sex-specific hormones and genetic susceptibility may play a role alongside differential exposure to risk factors between sexes (Sierra et al., 2016). Our results provide additional information that could support several hypotheses to explain similarities and differences between and within groups (by sexes) in their cancer burden from a contextual perspective.

Both in the female and male population, the high adherence to socioeconomically prosperous contexts were associated with increased cancer ASMR. Cancer epidemiology research has noted that the socioeconomic gradient associated with this disease differs by cancer types. A higher risk among socioeconomically advantaged people was observed for colon and breast cancers (Merletti et al., 2011), two major cancer-related cause of mortality in Argentina (accounting for $21.7 \%$ of total cancer-related deaths in 2015). Interestingly, Argentina presents colorectal and breast cancer mortality rates closer to those in more developed regions than in developing ones (Ferlay et al., 2013).

Behavioral factors linked to contextual circumstances may also explain our findings. It has been reported that around $30 \%$ of cancer deaths are due to a small group of modifiable risk factors, including elevated body mass index, reduced fruits and vegetables intake, and lack of physical activity (World Health Organization, 2017). It is possible that more socioeconomically developed contexts provide higher opportunities to make unhealthy food choices (easy access to processed foods at lower prices, fast-food outlets, hypermarkets and street foods) and involve increased use of motor vehicles, resulting in higher rates of sedentary lifestyle. Even so, rural poverty has also been previously associated to cancer burden in Argentina (Pou et al., 2017).

Reproductive behaviors, such as the parity and breastfeeding are well-documented risk factors for breast cancer (Lambertini et al., 2016). It should be noted that Argentina is one of the countries with the highest incidence rates of this cancer and also the lowest fertility rates in the Latin American region (with similar values to those in developed countries) (Di Sibio et al., 2016). It is possible that better access to medical and educational resources for family planning in socioeconomically more prosperous contexts, and diverse cultural values linked to gender roles in society may explain, in part, regional differences in the female population.

Comparison of the spatial distribution of "Plains region" pattern with the environment and socioeconomic QoLI mapping showed that the flat areas present mostly an unfavorable situation in terms of environmental conditions, and that the northeastern region presented the most unfavorable socioeconomic circumstances in the country. This area concentrates the worst health systemrelated indicators, including for example the screening coverage of cervical (Arrossi et al., 2008) and breast cancers (according to the 2009 National Survey of Risk Factors for Chronic Diseases). The observed association can be partially explained by the convergence of a context of higher poverty in the northeastern region (Pou et al., 2017) and probably a high exposure to carcinogenic chemicals (such as pesticides and others compounds coming from pollution) in the "Pampeana" region, which concentrates the highest population density in the country $(66 \%$ of all inhabitants in 2010$)$ and the greatest economic development based on agricultural industry.

In our results, female cancer mortality rates were higher in counties with more urban-related resources or cultural capital, suggesting that the educational level could be a potential determinant of the cancer mortality in women. Even so, education has been suggested as a social indicator of a person's skills to prevent health damages and to cope with illness through more appropriate pathways of care (Merletti et al., 2011). In Argentina, the female cancer mortality burden largely reflected the pattern of breast cancer mortality (accounting for $19.7 \%$ of overall cancer deaths in women) (Ferlay et al., 2013). Interestingly, the 2009 National Survey of Risk Factors for Chronic Diseases reports that the prevalence of mammography in the last two years in women with a complete secondary or higher education was around twice as high as in women who had not completed their primary education. We argue that urban contexts provide better and more easily accessible medical resources for cancer control and greater chances of education than less urbanized areas, all of which facilitates cancer detection in the female urban population. Although diagnosis of breast cancer at an early stage actually presuppose higher survival rates, it is plausible that high cancer incidence rates can drive high cancer mortality rates. Geographical differences in registration practices as well as in access to diverse treatments (considering their survival rates) (Woods et al., 2006) after breast cancer diagnosis may be relevant points in future studies.

Our results have shown that counties characterized by anthropic environmental exposures were associated with higher cancer mortality rates, only in the male population. Considering that the "Environments with anthropic exposures" pattern illustrates mainly the location of mining (in the north and northwest region) and other industrial sectors, we consider that this sex-related health difference lies in occupational differences. Occupation may reflect specific toxic environmental or work task exposures (Solar and Irwin, 2010). Work in the transport, industry and mining sectors is mainly done by men in Argentina, and it involves prolonged exposure to human carcinogenic chemical agents (Baan et al., 2009; Straif et al., 2009).

Ecological studies are susceptible to bias, such as the ecological fallacy. However, when an exposure is fairly common, ecologic designs are able to reveal the effects of such exposures (Lagiou et al., 2005). These studies are potentially useful for the formulation of etiological hypotheses and rapidly revealing inequities between social groups, which is valuable for public health objectives. With the emergence of the social determinant of health 
approach, this design acquires relevance again because it can incorporate upstream factors in the analysis (features that are intrinsic to places or contexts, which do not always have a counterpart at the individual level). Thus, interpretations of this study are valuable but should be cautious and refer to the aggregated (and not the individual) level.

It should be emphasized that the most socioeconomically prosperous region in Argentina is, in turn, the most urbanized. Paradoxically, urban poverty is hence concentrated in this area. Considering that other authors attribute a heavier burden of non-communicable diseases (including cancer) on the urban poverty (Friel et al., 2011), its effect should be clarified in future cancer research. In addition, we recognize that the selection of overall cancer as an outcome variable in the analysis is controversial, given that cancer involves many different diseases with different etiologies (Hiatt and Breen, 2008). However, cancer mortality in Argentina is concentrated in a few cancer sites (mainly lung, prostate and colorectal in men; breast, colorectal and lung in women) (Ferlay et al., 2013).

It is worth noting that the use of an integral quality of life index, which as far as we know is the only continuous quantitative index available in Argentina, constitutes a strength of our work. This index offers the advantage of integrating macro-environmental and socioeconomic aspects. In addition, we remark that our work obtained plausible results through a novelty methodological strategy (a principal component factor analysis coupled to mapping and multilevel modeling), considering the hierarchical structure of data (counties nested into provinces, simultaneously) and accounting the spatial variability in the estimation process. So, considering the increasing call for the use of multilevel methods and perspectives in epidemiologic cancer research (Zahnd and McLafferty, 2017), we highlight the applicability of our statistical approach in order to recognize geographical areas that have worse cancer outcome linked to disadvantageous socio-environmental features, which can be helpful from a public health perspective.

Concluding, this study provides valuable findings to characterize the socio-environmental context of Argentina and to suggest plausible hypotheses about the factors associated with the quality of life. These factors underlie the spatial pattern of female and male cancer mortality and they could explain health inequities in this country. In order to eliminate unfair and avoidable health differences between social groups in Argentina we propose to move the focus of attention away from the non-communicable diseases programs and policies (typically placed on individual risk factors) to the broad spectrum of social determinants of health. Both from the micro and macro level, it should be considered topics such as urban planning, occupational conditions, environmental quality, health-system efficiency and socioeconomic inequities.

\section{Funding Statement}

This work was supported by the National Science and Technology Agency [FONCyT, PICT 2012-1019] and the Science and Technical Department of the University of Córdoba [PID 1565/14].

\section{Acknowledgements}

We are especially grateful to the National Ministry of Health for providing the crude mortality-database for rate estimates. We would also like to thank Gabriela Díaz Cortez for her language assistance.

\section{References}

Alavanja MC, Ross MK, Bonner MR (2013). Increased cancer burden among pesticide applicators and others due to pesticide exposure. CA Cancer J Clin, 63, 120-42.

Arrossi S, Ramos S, Paolino M, Sankaranarayanan R (2008). Social inequality in Pap smear coverage: identifying under-users of cervical cancer screening in Argentina. Reprod Health Matters, 16, 50-8.

Baan R, Grosse Y, Straif K, et al (2009). A review of human carcinogens-Part F: chemical agents and related occupations. Lancet Oncol, 10, 1143-4.

Curado MP, de Souza DL (2014). Cancer burden in Latin America and the Caribbean. Ann Glob Health, 80, 370-7.

Díaz MdelP, Osella AR, Aballay LR, et al (2009). Cancer incidence pattern in Cordoba, Argentina. Eur J Cancer Prev, 18, 259-66.

Di Sibio A, Abriata G, Forman D, Sierra MS (2016). Female breast cancer in Central and South America. Cancer Epidemiol, 44, 110-20.

Ferlay J, Soerjomataram I, Ervik M, et al (2013). GLOBOCAN 2012 v1.0, Cancer incidence and mortality worldwide: International Agency for Research on Cancer CancerBase No. 11. http://globocan.iarc.fr (accessed 01.08.17).

Friel S, Akerman M, Hancock T, et al (2011). Addressing the social and environmental determinants of urban health equity: evidence for action and a research agenda. J Urban Health, 88, 860-74.

Ghislain I, Zikos E, Coens C, et al (2016). Health-related quality of life in locally advanced and metastatic breast cancer: methodological and clinical issues in randomised controlled trials. Lancet Oncol, 17, 294-304.

Hiatt RA, Breen N (2008). The social determinants of cancer: a challenge for transdisciplinary science. Am J Prev Med, 35, 141-50.

Johnson RA, Wichern DW (2001). Applied Multivariate Statistical Analysis, 5th ed. Prentice-Hall, New Jersey, p 361.

Lagiou P, Adami HO, Trichopoulos D (2005). Causality in cancer epidemiology. Eur J Epidemiol, 20, 565-74.

Lambertini M, Santoro L, Del Mastro L, et al (2016). Reproductive behaviors and risk of developing breast cancer according to tumor subtype: A systematic review and meta-analysis of epidemiological studies. Cancer Treat Rev, 49, 65-76.

Matos EL, Loria DI, Vilensky M (1994). Cancer mortality and poverty in Argentina: a geographical correlation study. Cancer Epidemiol Biomarkers Prev, 3, 213-8.

Merletti F, Galassi C, Spadea T (2011). The socioeconomic determinants of cancer. Environ Health, 10, 7.

Pou SA, Tumas N, Sánchez Soria D, Ortiz P, Díaz MP (2017). Large-scale societal factors and noncommunicable diseases: Urbanization, poverty and mortality spatial patterns in Argentina. Appl Geogr, 86, 32-40.

Rabe-Hesketh S, Skrondal A (2008). Multilevel and longitudinal modeling using stata, 2nd ed. Stata Press, College Station, TX, p 562.

Sierra MS, Soerjomataram I, Antoni S, et al (2016). Cancer patterns and trends in Central and South America. Cancer Epidemiol, 44, 23-42.

Asian Pacific Journal of Cancer Prevention, Vol $19 \mathbf{3 0 5 1}$ 
Solar O, Irwin A (2010). A conceptual framework for action on the social determinants of health. World Health Organization.

Straif K, Benbrahim-Tallaa L, Baan R, et al (2009). A review of human carcinogens-Part C: metals, arsenic, dusts, and fibres. Lancet Oncol, 10, 453-4.

Velázquez G (2016). A new index for study quality of life: combining socioeconomic and environmental indicators. In 'Indicators of Quality of Life in Latin America', Ed. Tonon G. Springer International Research, London, pp 57-77.

Wang SY, Hsu SH, Gross CP, et al (2016). Association between Time since Cancer Diagnosis and Health-Related Quality of Life: A Population-Level Analysis. Value Health, 19, 631-8.

Woods LM, Rachet B, Coleman MP (2006). Origins of socio-economic inequalities in cancer survival: a review. Ann Oncol, 17, 5-19.

World Health Organization (2017). Cancer. http://www.who. int/mediacentre/factsheets/fs297/en/ (accessed 01.08.17).

Zahnd WE, McLafferty SL (2017). Contextual effects and cancer outcomes in the United States: a systematic review of characteristics in multilevel analyses. Ann Epidemiol, 27, 739-48.

This work is licensed under a Creative Commons AttributionNon Commercial 4.0 International License. 